\title{
Krasnoselskii N-Tupled Fixed Point Theorem with Applications to Fractional Nonlinear Dynamical System
}

\author{
Tamer Nabil (iD ${ }^{1,2}$ \\ ${ }^{1}$ King Khalid University, College of Science, Department of Mathematics, P.O. Box 9004, 61413 Abha, Saudi Arabia \\ ${ }^{2}$ Suez Canal University, Faculty of Computers and Informatics, Department of Basic Science, Ismailia, Egypt \\ Correspondence should be addressed to Tamer Nabil; t_3bdelsadek@yahoo.com
}

Received 30 October 2018; Accepted 7 February 2019; Published 3 March 2019

Academic Editor: Sergey Shmarev

Copyright (c) 2019 Tamer Nabil. This is an open access article distributed under the Creative Commons Attribution License, which permits unrestricted use, distribution, and reproduction in any medium, provided the original work is properly cited.

In this paper, $\mathrm{N}$-tupled fixed point theorems for two monotone nondecreasing mappings in complete normed linear space are established. The extension of Krasnoseskii fixed point theorem for a version of N-tupled fixed point is given. Our theoretical results are applied to prove the existence of a mild solution of the system of $\mathrm{N}$-nonlinear fractional evolution equations. Finally, an example of a nonlinear fractional dynamical system is given to illustrate the results.

\section{Introduction}

It is outstanding that the fixed point theorem of Krasnoselskii [1] might be joined with Banach and Schauder's fixed point theorems. The sum of operators is obviously found in neutral functional equations and delay integral equations, which have been talked about widely in [2, 3], for instance. In 1964, Krasnoselskii studied the existence of fixed point for a sum of two operators, one being a continuous and compact and the other being a strict contraction. A lot of generalizations of Krasnoselskii's theorem have been done, mostly by relaxing the continuous and compact conditions and sometimes by withdrawing the requirement of the strict contraction or even both.

In 2006, Bhaskar and Lskshmikanthan [4] proposed new approach of the fixed point theorem which was called coupled fixed point. They gave some useful applications for mixed monotone operators.

In 2011, Luong and Thuan [5] extend the coupled fixed point approach to study the unique solution of the integral equations. Recently in 2013, Dhage [6] proposed new a version of the Krasnoselskii fixed point theorem and used it to prove the existence of the solution of nonlinear fractional differential equation.

More recently, in 2017, Yang et al. [7] proved the Krasnoselskii coupled fixed point theorem under some or certain conditions and used their results to study the existence of the solution of a nonlinear coupled system of fractional differential equations. Imdad et al. [8] in 2013, gave a new approach of the fixed point theory which was called N-tupled fixed point. This approach is useful to study a fixed point of mappings $F: \underbrace{X \times X \times \cdots \times X}_{n \text {-times }} \rightarrow X$, where $X$ is a nonempty set. In this paper, we extend the results of Yang et al. [7] to prove a generalized version of $\mathrm{N}$-tupled fixed point theorems. We give an application to the study existence of a solution of a system of nonlinear fractional differential evolution equations.

We organize the paper as follows: In Section 2, some basic definitions are introduced. In Section 3, Krasnoselskii $\mathrm{N}$-tupled fixed point ( KNTFP) is proved. In Section 4, the existence of the solution of a nonlinear $\mathrm{N}$-system of fractional evolution differential equations is considered. In Section 5, we give an example of a nonlinear fractional dynamical system. Finally, in Section 6 the conclusion is presented.

\section{Preliminaries}

Let $X \neq \phi$ and $F: X \times X \longrightarrow X$ be a mapping. A point $(x, y) \in X \times X$ is said to be a coupled fixed point of $F$ in $X \times X$ if $F(x, y)=x$ and $F(y, x)=y$. Throughout this paper, let $E$ be a nonempty set and $(E, \leq,\|\|$.$) be a partially ordered normed$ 
linear space. If $Q: E \longrightarrow E$ is a mapping. Then $Q$ is said to be monotone nondecreasing if $a \leq b$ implies $Q(a) \leq Q(b)$, for all $a, b \in E$. Two elements $a, b \in E$ are said to be comparable if $a \leq b$ or $b \leq a$. If $C$ is a nonempty subset of $E, C$ is said to be a chain if each two elements $a, b \in C$ are comparable. We recall the following definitions which are given in $[6,7]$.

Definition 1. Let $Q: E \longrightarrow E$ be a mapping. $Q$ is called partially compact if for each chain $C$ subset of $E, Q(C)$ is a relatively compact subset of $E$.

Definition 2. Let $Q: E \longrightarrow E$ be a mapping. Given an element $a \in E$, define the orbit $\Upsilon(a ; Q)$ as

$$
\Upsilon(a ; Q)=\left\{a, Q a, Q^{2} a, Q^{3} a, \ldots, Q^{n} a, \ldots\right\} .
$$

If for any sequence $\left\{a_{n}\right\} \in \Upsilon(a ; Q)$ such that $a_{n} \longrightarrow a^{*}$ as $n \longrightarrow \infty$ implies $Q a_{n} \longrightarrow Q a^{*}$, for each $a \in E$, then $Q$ is called $\Upsilon a$ - orbitally continuous in $E$. Furthermore, $(E, \leq,\|\|$. is said to be $Y$ - orbitally complete if each sequence $\left\{a_{n}\right\} \in$ $\Upsilon(a ; Q)$ converges to an element $a^{*} \in E$.

Definition 3. A mapping $\phi: E \longrightarrow E$ is said to be $D$ - function if it is upper semicontinuous and monotone nondecreasing such that $\phi(0)=0$.

Definition 4. A mapping $Q: E \longrightarrow E$ is called partially nonlinear $D$ - contraction in $E$, if for each comparable elements $a, b \in E$, there exists a $D$ - function $\phi: \mathfrak{R}^{+} \longrightarrow \mathfrak{R}^{+}$ such that the following conditions are satisfied:

(i) $\|Q a-Q b\| \leq \phi(\|a-b\|)$,

(ii) $\phi(r)<r$ for all $r>0$.

Definition 5. Let $(E, \leq,\|\|$.$) be a \Upsilon$ - orbitally complete linear space. The positive cone $K \in E$ is defined as $K=\{x \in E: x \geq$ $0\}$.

The following theorem was given in [7] and will be used as a tool to prove the main results.

Theorem 6. Let $E$ be a partially ordered $\Upsilon$-orbitally complete normed linear space and $K$ be the positive cone of $E$. Let $K$ be normal and $C$ be a nonempty closed subset of $E$. Consider the two monotone nondecreasing mappings $P, Q: C \longrightarrow C$ such that the following conditions are satisfied:

(1) $P$ is $\Upsilon$ - orbitally continuous and a partially nonlinear $D$ - contraction,

(2) $Q$ is $\Upsilon$ - orbitally continuous and partially compact,

(3) there xists an element $a \in C$ such that $a \leq P a+Q y$ for all $y \in C$, bound.

(4) every pair of elements in C has an upper and lower

Then $F(x, y)=P x+Q y$ has a coupled fixed point in $E \times E$.

Now, we recall the definition about the fractional derivative; for more detail about the fractional calculus, please see $[9,10]$. The fractional derivative is defined via the fractional integral operator. So, we will start by the definition of the fractional integral operator. The fractional integral operator of order $\alpha(>0) \in(0, \infty)$ of a function $g \in L^{1}([0, b], E)$ such that $b>0$ is defined as

$$
I^{\alpha} g(t)=\int_{0}^{t} \frac{(t-s)^{\alpha-1}}{\Gamma(\alpha)} g(s) d s .
$$

Also, the Riemann-Liouville derivative of order $\alpha(>0)$ is defined as

$$
{ }^{R} D^{\alpha} g(t)=D^{m}\left(I^{m-\alpha} g(t)\right),
$$

where $m=\lceil\alpha\rceil$ and $D=d / d t$.

The Caputo fractional derivative of order $\alpha(>0)$ is denoted by $D_{*}^{\alpha}$ and is defined as

$$
D_{*}^{\alpha} g(t)=I^{n-\alpha}\left(D^{n} g(t)\right),
$$

where $n=\lceil\alpha\rceil, g \in L^{1}([0, b], E)$ such that $b>0$ and $t>0[11]$.

\section{Krasnoselshii N-Tupled Fixed Point Theorem}

In this section we give the main $\mathrm{N}$-tupled fixed point theorem which is used in the proposed applications. Consider $E^{n}=$ $\underbrace{E \times E \times \cdots \times E}_{n \text {-times }}$ and define the two operations (sum and scaler multiplication in $\left.E^{n}\right)$ as follows: if $W=\left(w_{1}, w_{2}, \ldots, w_{n}\right), V=$ $\left(v_{1}, v_{2}, \ldots, v_{n}\right) \in E^{n}$, and $\lambda \in R$ then, we have that

$$
\begin{aligned}
W+V & =\left(w_{1}+v_{1}, w_{2}+v_{2}, \ldots, w_{n}+v_{n}\right) \\
\lambda V & =\left(\lambda v_{1}, \lambda v_{2}, \ldots, \lambda v_{n}\right)
\end{aligned}
$$

Therefore, the positive cone in $E^{n}$ can be defined as

$$
\begin{aligned}
& K_{E^{n}} \\
& \quad=\left\{W=\left(w_{1}, w_{2}, \ldots, w_{n}\right) \in E^{n}: w_{1}, w_{2}, \ldots, w_{n} \geq 0\right\} .
\end{aligned}
$$

Also the norm in $E^{n}$ can be defined as

$$
\begin{aligned}
\|W\|_{E^{n}} & =\left\|\left(w_{1}, w_{2}, \ldots, w_{n}\right)\right\| \\
& =\left\|w_{1}\right\|+\left\|w_{2}\right\|+\cdots+\left\|w_{n}\right\|,
\end{aligned}
$$

where $\|$.$\| is the norm defined on E$. It is easy to prove that $\left(E^{n},\|\cdot\|_{E^{n}}\right)$ is partially ordered normed linear space with order relation $\leq$ induced by $K_{E^{n}}$. If $D$ is a nonempty closed subset of $E$, then $\underbrace{D \times D \times \cdots \times D}_{n-\text { times }}$ is also nonempty closed subset of $E^{n}$. On the other hand, if $K$ is normal cone, the $K_{E^{n}}$ is normal.

Let $Q: E^{n} \longrightarrow E$. The point $\left(x_{1}, x_{2}, \ldots, x_{n}\right) \in E^{n}$ is said to be $\mathrm{N}$-tupled fixed point of $Q$ if and only if

$$
\begin{gathered}
x_{1}=Q\left(x_{1}, x_{2}, \ldots, x_{n}\right) \\
x_{2}=Q\left(x_{2}, x_{3}, \ldots, x_{n}, x_{1}\right) \\
\vdots \\
x_{n}=Q\left(x_{n}, x_{1}, \ldots, x_{n-1}\right)
\end{gathered}
$$

Now, we prove the main fixed point theorem. 
Theorem 7. Let $E$ be a partially ordered $\Upsilon$ - orbitally complete normed linear space and $K$ be the positive cone of $E$. Let $K$ be normal and $D$ be a nonempty closed subset of $E$. Consider $T_{1}, T_{2}, \ldots, T_{n}: D \longrightarrow D$ are $n$ monotone nondecreasing mappings such that the following are satisfied:

(1) $T_{1}$ is $\Upsilon$-orbitally continuous and a partially nonlinear $D$ - contraction,

(2) $T_{2}, \ldots, T_{n}$ are $\Upsilon$ - orbitally continuous and a partially compact,

(3) there exists an element $a \in D$ such that $a \leq T_{1} a+T_{2} y_{2}+$ $T_{3} y_{3}+\cdots+T_{n} y_{n}$ for all $y_{2}, y_{3}, \ldots, y_{n} \in D$,

(4) every pair of elements in D has an upper and lower bounded.

Then $Q\left(x_{1}, x_{2}, \ldots, x_{n}\right)=T_{1} x_{1}+T_{2} x_{2}+\cdots+T_{n} x_{n}$ has a $N$-tupled fixed point in $E^{n}$.

Proof. According to the fact that $(E, \leq,\|\|$.$) is a partially$ ordered $Y$ - complete normed linear space and $K$ is normal, then $\left(E^{n}, \leq,\|\cdot\|_{E^{n}}\right)$ is also a partially ordered $\Upsilon$ - complete normed linear space and $K_{E^{n}}$ is normal. Define $D^{n}=$ $\underbrace{D \times D \times \cdots \times D}_{n-\text { times }}$, then $D^{n}$ is closed subset of $E^{n}$. Consider $P_{1}, P_{2}, \ldots, P_{n}: D^{n} \longrightarrow D^{n}$ such that, for $u=\left(x_{1}, x_{2}, \ldots, x_{n}\right) \in$ $D^{n}$,

$$
\begin{gathered}
P_{1} u=\left(T_{1} x_{1}, T_{1} x_{2}, \ldots, T_{1} x_{n}\right) \\
P_{2} u=\left(T_{2} x_{2}, T_{2} x_{3}, \ldots, T_{2} x_{n}, T_{2} x_{1}\right) \\
\vdots \\
P_{n} u=\left(T_{n} x_{n}, T_{n} x_{1}, T_{n} x_{2}, \ldots, T_{n} x_{n-1}\right)
\end{gathered}
$$

Therefore, if we prove that the operator equation $u=P_{1} u+$ $P_{2} u+\cdots+P_{n} u$ has a solution $u=\left(x_{1}, x_{2}, \ldots, x_{n}\right) \in D^{n}$, then we have that

$$
\begin{aligned}
\left(x_{1}, x_{2}, \ldots, x_{n}\right)= & \left(T_{1} x_{1}, T_{1} x_{2}, \ldots, T_{1} x_{n}\right) \\
& +\left(T_{2} x_{2}, T_{2} x_{3}, \ldots, T_{2} x_{n}, T_{2} x_{1}\right) \\
& +\cdots \\
& +\left(T_{n} x_{n}, T_{n} x_{1}, T_{n} x_{2}, \ldots, T_{n} x_{n-1}\right) .
\end{aligned}
$$

This implies that $\left(x_{1}, x_{2}, \ldots, x_{n}\right)$ is a $\mathrm{N}$-tupled fixed point of $Q$ in $E^{n}$. Our proof of the theorem is divided into 4 steps.

Step 1. $P_{1}$ is $\Upsilon$ - orbitally continuous and $\left\|P_{1} W-P_{2} V\right\|_{E^{n}} \leq$ $\|W-V\|_{E^{n}}$, for all comparable $W \neq V \in D^{n}$. Since $T_{1}$ is $\Upsilon-$ orbitally continuous then by the definition of $P_{1}$ we get that $P_{1}$ is $\Upsilon$ - orbitally continuous.

Let $W \neq V \in D^{n}$ be comparable elements; we have that

$$
\begin{aligned}
& \left\|P_{1} W-P_{2} V\right\|_{E^{n}}=\|\left(T_{1} w_{1}, T_{1} w_{2}, \ldots, T_{1} w_{n}\right) \\
& \quad-\left(T_{1} v_{1}, T_{1} v_{2}, \ldots, T_{1} v_{n}\right)\left\|_{E^{n}} \leq\right\| T_{1} w_{1}-T_{1} v_{1} \|+\cdots \\
& \quad+\left\|T_{1} w_{n}-T_{1} v_{n}\right\| \leq \phi\left(\left\|w_{1}-v_{1}\right\|\right)+\cdots \\
& \quad+\phi\left(\left\|w_{n}-v_{n}\right\|\right) \leq\left\|w_{1}-v_{1}\right\|+\cdots+\left\|w_{n}-v_{n}\right\| \\
& \quad=\|W-V\|_{E^{n}},
\end{aligned}
$$

Step 2. $P_{2}, P_{3}, \ldots, P_{n}$ are $\Upsilon$-orbitally continuous and partially compact.

Since $T_{2}, \ldots, T_{n}$ are $\Upsilon$-orbitally continuous, it is easy to prove that $P_{2}, \ldots, P_{n}$ are $\Upsilon$ - orbitally continuous. Consider $C \subset D$ is bounded chain. According to the fact that $T_{2}$ is partially compact in $D$, then we get that $T_{2}(C)$ is equicontinuous and uniformly bounded in $D$. Let $C^{n}=$ $C \times C \times \cdots \times C$, then $C^{n} \subset D^{n}$ and $C^{n}$ is bounded chain n-times

in $D^{n}$. Now, we prove that $P_{2}\left(C^{n}\right)$ is equicontinuous and uniformly bounded in $D^{n}$. Since $P_{2}(C) \subset D$ is uniformly bounded, there is a constant $M_{2}>0$ satisfying $\left\|T_{2} z\right\| \leq \varepsilon$ for all $z \in C$. For any $Z \in P_{2}\left(C^{n}\right)$, we can find $y_{1}, y_{2}, \ldots, y_{n} \in C$ such that $u=\left(y_{1}, y_{2}, \ldots, y_{n}\right) \in C^{n}$ and $Z=P_{2} u$. Therefore, we have that

$$
\begin{aligned}
\|Z\|_{E^{n}} & =\left\|P_{2} u\right\|_{E^{n}}=\left\|\left(T_{2} y_{2}, T_{2} y_{3}, \ldots, T_{2} y_{n}, T_{2} y_{1}\right)\right\|_{E^{n}} \\
& =\left\|T_{2} y_{2}\right\|+\left\|T_{2} y_{3}\right\|+\cdots+\left\|T_{2} y_{n}\right\|+\left\|T_{2} y_{1}\right\| \\
& \leq n \varepsilon .
\end{aligned}
$$

Thus, $P_{2}\left(C^{n}\right)$ is uniformly bounded in $D^{n}$. By doing the same steps we get that $P_{3}, \ldots, P_{n}$ are uniformly bounded in $D^{n}$. According to the fact that $T_{2}(C)$ is equicontinuous in $D$, for any $z \in C$ and $t_{2}>t_{1}$, we have that $\left\|\left(T_{2} z\right)\left(t_{2}\right)-\left(T_{2} z\right)\left(t_{1}\right)\right\| \longrightarrow$ 0 , as $t_{2}-t_{1} \longrightarrow 0$. Thus, for any $\mathrm{Z}=P_{2} u \in P_{2}\left(C^{n}\right)$, where $t_{2}-t_{1} \longrightarrow 0$, we get the following:

$$
\begin{aligned}
& \left.\left\|Z\left(t_{2}\right)-Z\left(t_{1}\right)\right\|_{E^{n}}=\|\left(P_{2} u\right)\left(t_{2}\right)-\left(P_{2} u\right)\left(t_{1}\right)\right) \|_{E^{n}} \\
& =\left\|\left(T_{2} y_{2}\right)\left(t_{2}\right)-\left(T_{2} y_{2}\right)\left(t_{1}\right)\right\| \\
& \quad+\left\|\left(T_{2} y_{3}\right)\left(t_{2}\right)-\left(T_{2} y_{3}\right)\left(t_{1}\right)\right\|+\cdots \\
& \quad+\left\|\left(T_{2} y_{1}\right)\left(t_{2}\right)-\left(T_{2} y_{1}\right)\left(t_{1}\right)\right\| \longrightarrow 0 .
\end{aligned}
$$

Thus, $P_{2}\left(C^{n}\right)$ is equicontinuous in $D_{n}$. Therefore, $P_{2}\left(C^{n}\right)$ is relatively compact in $D^{n}$. Hence, $P_{2}: D^{n} \longrightarrow D^{n}$ is partially compact. By doing the same steps, we get that $P_{3}, P_{3}, \ldots, P_{n}$ : $D^{n} \longrightarrow D^{n}$ are partially compact.

Step 3. There is an element $u_{0} \in D$ satisfying: $u_{0} \leq P_{1} u_{0}+$ $P_{2} u+\cdots+P_{n} u$ for all $u \in D^{n}$

Let $u_{0}=(a, a, \ldots, a)$ and $u=\left(x_{1}, x_{2}, \ldots, x_{n}\right) \in D^{n}$. By condition (3) in the theorem, we get that

$$
\begin{aligned}
P_{1} u_{0}+ & P_{2} u+\cdots+P_{n} u \\
= & \left(T_{1} a, T_{1} a, \ldots, T_{1} a\right)+\left(T_{2} x_{2}, T_{2} x_{3}, \ldots, T_{2} x_{1}\right) \\
& +\cdots+\left(T_{n} x_{n}, T_{n} x_{1}, \ldots, T_{n} x_{n-1}\right) \geq(a, a, \ldots, a) .
\end{aligned}
$$

Step 4. Every pair of elements in $D^{n}$ has an upper bound and lower bound. For every pair of elements $W=$ $\left(w_{1}, w_{2}, \ldots, w_{n}\right), V=\left(v_{1}, v_{2}, \ldots, v_{n}\right) \in D^{n}$, from condition 
(4) of the theorem, there exist $a_{1}, a_{2}, \ldots, a_{n}$ and $b_{1}, b_{2}, \ldots, b_{n}$ such that, for all $i=1,2, \ldots, n$, we have that

$$
\begin{aligned}
& a_{i} \leq w_{i}, \\
& a_{i} \leq v_{i}, \\
& b_{i} \geq w_{i}, \\
& b_{i} \geq v_{i} .
\end{aligned}
$$

Hence, we find that

$$
\begin{aligned}
& \left(a_{1}, a_{2}, \ldots, a_{n}\right) \leq\left(w_{1}, w_{2}, \ldots, w_{n}\right) \leq\left(b_{1}, b_{2}, \ldots, b_{n}\right), \\
& \left(a_{1}, a_{2}, \ldots, a_{n}\right) \leq\left(v_{1}, v_{2}, \ldots, v_{n}\right) \leq\left(b_{1}, b_{2}, \ldots, b_{n}\right) .
\end{aligned}
$$

Thus, we get that every pair $W, V \in D^{n}$ has an upper and lower bound.

Define $B: D^{n} \longrightarrow D^{n}$ as $B U=P_{2} U+P_{3} U+\cdots+P_{n} U$, for all $U \in D^{n}$. Thus $B$ is $\Upsilon$ - orbitally continuous and a partially compact. Also there exists an element $u_{0} \in D^{n}$ such that $u_{0} \leq$ $P_{1} u_{0}+B V$ for all $V \in D^{n}$. Hence $U=P_{1} U+B U$ has a solution in $E^{n}$. Then if the solution is $\left(x_{1}, x_{2}, \ldots, x_{n}\right)$, we get that

$$
\begin{aligned}
\left(x_{1}, x_{2}, \ldots, x_{n}\right)= & \left(T_{1} x_{1}, T_{1} x_{2}, \ldots, T_{1} x_{n}\right) \\
& +\left(T_{2} x_{2}, T_{2} x_{3}, \ldots, T_{2} x_{n}, T_{2} x_{1}\right) \\
& +\cdots \\
& +\left(T_{n} x_{n}, T_{n} x_{1}, T_{n} x_{2}, \ldots, T_{n} x_{n-1}\right) .
\end{aligned}
$$

Therefore, we have that

$$
\begin{gathered}
x_{1}=T_{1} x_{1}+T_{2} x_{2}+\cdots+T_{n} x_{n}=Q\left(x_{1}, x_{2}, \ldots, x_{n}\right) \\
x_{2}=T_{1} x_{2}+T_{2} x_{3}+\cdots+T_{n} x_{1}=Q\left(x_{2}, x_{3}, \ldots, x_{1}\right) \\
\vdots \\
x_{n}=T_{1} x_{n}+T_{2} x_{1}+\cdots+T_{n} x_{n-1} \\
=Q\left(x_{n}, x_{1}, \ldots, x_{n-1}\right)
\end{gathered}
$$

Hence $Q\left(x_{1}, x_{2}, \ldots, x_{n}\right)$ has a N-tupled fixed point. By changing condition (3) due to the fact that there exists an element $a \in D$ such that $a \geq T_{1} a+T_{2} y_{2}+T_{3} y_{3}+\cdots+T_{n} y_{n}$ for all $y_{2}, y_{3}, \ldots, y_{n} \in D$, the theoretical results are also correct.

Theorem 8. Let E be a partially ordered $\Upsilon$ - orbitally complete normed linear space and $K$ be the positive cone of $E$. Let $K$ be normal and $D$ be a nonempty closed subset of E. Consider $T_{1}, T_{2}, \ldots, T_{n}: D \longrightarrow D$ are $n$ monotone nondecreasing mappings such that the following are satisfied:

(1) $T_{1}$ is $\Upsilon$-orbitally continuous and a partially nonlinear $D$ - contraction,

(2) $T_{2}, \ldots, T_{n}$ are $\Upsilon$ - orbitally continuous and a partially compact,

(3) there exists an element $a \in D$ such that $a \geq T_{1} a+T_{2} y_{2}+$ $T_{3} y_{3}+\cdots+T_{n} y_{n}$ for all $y_{2}, y_{3}, \ldots, y_{n} \in D$,

(4) every pair of elements in $D$ has an upper and lower bounded.

Then $Q\left(x_{1}, x_{2}, \ldots, x_{n}\right)=T_{1} x_{1}+T_{2} x_{2}+\cdots+T_{n} x_{n}$ has a $N$-tupled fixed point in $E^{n}$.

\section{Existence of Mild Solution of the System of N- Fractional Evolution Equations}

Let $\widetilde{K}$ be the positive convex cone of $\Upsilon$ - orbitally Banach space $(X,\|\|$.$) . Suppose that \widetilde{K}$ is normal. It is clear that $X$ is partially order by the poset $\leq$ induced by $\widetilde{K}$. In this section, we prove the existence of mild solution of the system of $\mathrm{N}$ fractional evolution evolution equations:

$$
\begin{aligned}
& D_{*}^{\alpha} x_{1}(t)+A x_{1}(t)=f_{1}\left(t, x_{1}(t)\right)+f_{2}\left(t, x_{2}(t)\right)+\cdots \\
& \quad+f_{n}\left(t, x_{n}(t)\right), \\
& D_{*}^{\alpha} x_{2}(t)+A x_{2}(t)=f_{1}\left(t, x_{2}(t)\right)+f_{2}\left(t, x_{3}(t)\right)+\cdots \\
& \quad+f_{n}\left(t, x_{1}(t)\right), \\
& \quad \vdots \\
& \left.\quad+f_{n}\left(t, x_{n-1}(t)\right)\right), \\
& D_{*}^{\alpha} x_{n}(t)+A x_{n}(t)=f_{1}\left(t, x_{n}(t)\right)+f_{2}\left(t, x_{1}(t)\right)+\cdots \\
& x_{i}(0)=\zeta_{0} \in X, \quad \forall i=1,2, \ldots, n, \\
& \alpha \in(0,1), \quad t \in[0, b], \quad b>0,
\end{aligned}
$$

where $-A$ generates a $C_{0}-$ semigroup $\{S(t): t \geq 0\}$ of uniformly linear and bounded operators in $X$. Also, $f_{1}, f_{2}, \ldots, f_{n}$ are given functions. According to the fact that $S(t)$ are bounded, then there exists $d \in(0, \infty)$ such that $\|S(t)\| \leq d$ for all $t \geq 0$.

Define two families of operator $\left\{\Psi_{\alpha}(t): t \geq 0\right\}$ and $\left\{\Phi_{\alpha}(t): t \geq 0\right\}$

$$
\begin{aligned}
& \Psi_{\alpha}(t) x=\int_{0}^{\infty} \eta_{\alpha}(s) S\left(t^{\alpha} s\right) x d s, \\
& \Phi_{\alpha}(t) x=\alpha \int_{0}^{\infty} s \eta_{\alpha}(s) S\left(t^{\alpha} s\right) x d s
\end{aligned}
$$

where $\alpha \in(0,1)$ and

$$
\begin{aligned}
& \eta_{\alpha}(s)=\frac{1}{\alpha} s^{-1-1 / \alpha} \gamma_{\alpha}\left(s^{-1 / \alpha}\right), \\
& \gamma_{\alpha}(s)=\frac{1}{\pi} \sum_{n=1}^{\infty}(-1)^{n-1} s^{-\alpha n-1} \frac{\Gamma(n \alpha+1)}{n !} \sin (n \pi \alpha), \\
& \quad s \in(0, \infty) .
\end{aligned}
$$

According to $[12,13]$, the family of operators $\left\{\Psi_{\alpha}(t): t \geq 0\right\}$ and $\left\{\Phi_{\alpha}(t): t \geq 0\right\}$ has the following properties:

$(\operatorname{Pr} 1)$ For any $x \in X$ and $t \geq 0$ fixed, we have that

$$
\begin{aligned}
& \left\|\Psi_{\alpha}(t) x\right\| \leq d\|x\|, \\
& \left\|\Phi_{\alpha}(t) x\right\| \leq \frac{d}{\Gamma(\alpha)}\|x\|,
\end{aligned}
$$

$(\operatorname{Pr} 2)\{S(t): t \geq 0\}$ is an equicontinuous semigroup which implies that $\left\{\Phi_{\alpha}(t): t \geq 0\right\}$ is equicontinuous in $X$, 
(Pr3) $\{S(t): t \geq 0\}$ is positive $C_{0}-$ semigroup which implies that $\left\{\Psi_{\alpha}(t): t \geq 0\right\}$ and $\left\{\Phi_{\alpha}(t): t \geq 0\right\}$ are positive operators.

Define $C_{[0, b]}(X)=\{f:[0, b] \longrightarrow X: f$ is continuous $\}$ and $\widetilde{K}_{C}=\left\{x \in C_{[0, b]}(X): x(t) \in \widetilde{K}, t \in[0, b]\right\}$. It is easy to show $C_{[0, b]}(X)$ is a partially order $\Upsilon$ - orbitally complete normed space with the norm: $\|x\|_{C_{[0, b]}}=\sup _{t \geq 0}\|x(t)\|$, and a poset $\leq$ induced by $\widetilde{K}_{C}$. It is easy to show that $\widetilde{K}_{C}$ is normal because $\widetilde{K}$ is normal.

Let $E=C_{[0, b]}(X)$ and $K=\widetilde{K}_{C}$. Define, $n-$ operators $T_{1}, T_{2}, \ldots, T_{n}: E \longrightarrow E$ as

$$
\begin{aligned}
& \left(T_{1} x\right)(t)=\Psi_{\alpha}(t) \xi_{0} \\
& +\int_{o}^{t}(t-s)^{\alpha-1} \Phi_{\alpha}(t-s) f_{1}(s, x(s)) d s, \\
& t \in[0, b], \\
& \left(T_{2} x\right)(t)=\int_{o}^{t}(t-s)^{\alpha-1} \Phi_{\alpha}(t-s) f_{2}(s, x(s)) d s, \\
& t \in[0, b], \\
& \left(T_{3} x\right)(t)=\int_{o}^{t}(t-s)^{\alpha-1} \Phi_{\alpha}(t-s) f_{3}(s, x(s)) d s, \\
& t \in[0, b], \\
& \left(T_{n} x\right)(t)=\int_{o}^{t}(t-s)^{\alpha-1} \Phi_{\alpha}(t-s) f_{n}(s, x(s)) d s, \\
& t \in[0, b] .
\end{aligned}
$$

Then we can say that an element $\left(x_{1}, x_{2}, \ldots, x_{n}\right) \in E_{n}$ is a N-tupled mild solution of the system (19) if and only if $\left(x_{1}, x_{2}, \ldots, x_{n}\right)$ is the solution of the system of the operator equations:

$$
\begin{gathered}
x_{1}(t)=\left(T_{1} x_{1}\right)(t)+\left(T_{2} x_{2}\right)(t)+\cdots+\left(T_{n} x_{n}\right)(t), \\
t \in[0, b], \\
x_{2}(t)=\left(T_{1} x_{2}\right)(t)+\left(T_{2} x_{3}\right)(t)+\cdots+\left(T_{n} x_{1}\right)(t), \\
t \in[0, b], \\
x_{n}(t)=\left(T_{1} x_{n}\right)(t)+\left(T_{2} x_{1}\right)(t)+\cdots+\left(T_{n} x_{n+1}\right)(t), \\
t \in[0, b] .
\end{gathered}
$$

We will apply Theorem 7 to prove that the system of operator equations (24) has a $\mathrm{N}$-tupled fixed point in $E_{n}$ under the following important conditions:

$\left(C_{1}\right)$ The positive $C_{0}-$ semigroups $\{S(t): t \geq 0\}$ is equicontinuous.
$\left(C_{2}\right)$ The function $f_{1}:[0, b] \times X \longrightarrow X$ is continuous in $X$ for all $t \in[0, b]$ and there exist a constant $\varrho \in R$ with $0<\varrho<\Gamma(\alpha+1) / d b^{\alpha}$ and a $D$ - function $\phi$ : $[0, \infty) \longrightarrow[0, \infty)$ and satisfied the fact that $\phi(t)<r$ for any $r \in(0, \infty)$ and

$$
0 \leq f_{1}\left(t, e_{1}\right)-f_{1}\left(t, e_{2}\right) \leq \varrho \phi\left(\left\|e_{1}-e_{2}\right\|\right),
$$

for all $e_{1}, e_{2}\left(e_{1} \geq e_{2}\right) \in X$ and $t \in[0, b]$.

$\left(C_{3}\right)$ The functions $f_{2}(t, x), f_{3}(t, x), \ldots, f_{n}(t, x)$ :

$[0, b] \times X \longrightarrow X$ are continuous, nondecreasing, and bounded in $x$.

$\left(C_{4}\right)$ There exists an element $\tilde{x} \in E$ which satisfies the fact that

$$
\begin{aligned}
D_{*}^{\alpha} \tilde{x}(t)+A \tilde{x}(t) \leq & \left.f_{1}(t, \tilde{x})\right)+f_{2}\left(t, y_{2}(t)\right)+\cdots \\
& +f_{n}\left(t, y_{n}(t)\right), \\
& \forall y_{2}, \ldots, y_{n} \in E,
\end{aligned}
$$

$$
\begin{aligned}
& \tilde{x} \leq \xi_{0} \in X, \\
& \alpha \in(0,1), \quad t \in[0, b], b>0 .
\end{aligned}
$$

Theorem 9. If conditions $\left(C_{1}\right),\left(C_{2}\right),\left(C_{3}\right)$, and $\left(C_{4}\right)$ are satisfied, the system of fractional evolution equations (19) has a $N$-tupled mild solution on $[0, b]$.

Proof. Since condition $\left(C_{3}\right)$ holds, then $f_{2}(t, x), f_{3}(t, x), \ldots, f_{n}(t, x)$ are bound for all $t \in[0, b]$, and there are constants $N_{2}, N_{3}, \ldots N_{n}$ such that, for all $t \in[0, b]$, we get that

$$
\begin{gathered}
\left\|f_{2}(t, x)\right\| \leq N_{2}, \\
\left\|f_{3}(t, x)\right\| \leq N_{3}, \\
\vdots \\
\left\|f_{n}(t, x)\right\| \leq N_{n},
\end{gathered}
$$

$\forall x \in X$.

For all $i=2, \ldots, n$, we have that

$$
\begin{gathered}
\tilde{r} \geq \max \left\{\frac{d N_{i} b^{\alpha}}{\Gamma(\alpha+1)},\left(1-\frac{d \varrho b^{\alpha}}{\Gamma(\alpha+1)}\right)^{-1}\right. \\
\left.\cdot\left(d\left\|\xi_{0}\right\|+\frac{d \widetilde{G} b^{\alpha}}{\Gamma(\alpha+1)}\right)\right\}+1,
\end{gathered}
$$

where $\widetilde{G}=\sup \left\{\left\|f_{1}(t, 0)\right\|: t \in[0, b]\right\}$. We can define the following open balls in $E$ as

$$
B\left(x_{0}, r\right)=\left\{x \in E:\left\|x_{0}-x\right\|_{C_{[0, b]}}<r\right\}
$$

where $r=\left\|x_{0}\right\|_{C_{[0, b]}}+\widetilde{r}$. Define $D=\bar{B}\left(x_{0}, r\right)$. It is clear that $D$ is bounded and closed subset in $E$. Now we apply 3 steps to prove the theorem.

Step 1 . The operator $T_{1}: D \longrightarrow D$ is $\Upsilon$-orbitally continues and a partially nonlinear $D$ - contraction in $E$. 
Since condition $\left(C_{2}\right)$ holds, we get that, for any $x \in E$ with $\|x\|_{C_{[0, b]}} \leq \tilde{r}$, we have

$$
\begin{aligned}
& \left\|\left(T_{1} x\right) t\right\| \leq d\left\|\xi_{0}\right\|+\frac{d}{\Gamma(\alpha)} \int_{0}^{t}(s-t)^{\alpha-1} \\
& \cdot\left[\left\|f_{1}(s, x(s))-f_{1}(s, 0)\right\|+\left\|f_{1}(s, 0)\right\|\right] d s \\
& \leq d\left\|\xi_{0}\right\|+\frac{d}{\Gamma(\alpha)} \int_{0}^{t}(s-t)^{\alpha-1} \\
& \cdot\left[\varrho \phi\left(\|x\|_{C_{[0, b]}}\right)+\widetilde{G}\right] d s \leq d\left\|\xi_{0}\right\|+\frac{d \varrho b^{\alpha}}{\Gamma(\alpha+1)} \widetilde{r} \\
& +\frac{d \widetilde{G} b^{\alpha}}{\Gamma(\alpha+1)} \leq \widetilde{r} .
\end{aligned}
$$

By taking the sup, we get that $\left\|\left(T_{1} x\right) t\right\|_{C_{[0, b]}} \leq \widetilde{r}$ for all $x \in E$, such that $\|(x)\|_{C_{[0, b]}} \leq \widetilde{r}$. Therefore, we have the following:

$$
\begin{aligned}
\left\|x_{0}-T_{1} x\right\|_{C_{[0, b]}} & \leq\left\|x_{0}\right\|_{C_{[0, b]}}+\left\|T_{1} x\right\|_{C_{[0, b]}} \\
& \leq\left\|x_{0}\right\|_{C_{[0, b]}}+\tilde{r}=r_{1} .
\end{aligned}
$$

Hence, $T_{1}$ is a function from $D$ to $D$. According to the fact that $\{S(t): t \geq 0\}$ is positive $C_{0}$ - semigroup and the properties $(\operatorname{Pr} 1),(\operatorname{Pr} 2)$, and $(\operatorname{Pr} 3)$, it is provided that $T_{1}: D \longrightarrow D$ is nondecreasing. Let $\left\{x_{m}\right\} \in \Upsilon\left(x ; T_{1}\right)$ for any $x \in D$ such that $x_{m} \longrightarrow \widehat{x}$ as $m \longrightarrow \infty$. Thus by condition $\left(C_{2}\right)$, we get that $T_{1}$ is continuous in $X$ for all $t \in[0, b]$, and therefore we have the following:

$$
\begin{aligned}
\lim _{m \rightarrow \infty} & \left(T_{1} x_{m}\right)(t) \\
= & \Psi_{\alpha}(t) \xi_{0} \\
& \quad+\int_{0}^{t}(t-s)^{\alpha-1} \Phi_{\alpha}(t-s) \lim _{m \rightarrow \infty} f_{1}\left(s, x_{m}(s)\right) d s \\
= & \Psi_{\alpha}(t) \xi_{0}+\int_{0}^{t}(t-s)^{\alpha-1} \Phi_{\alpha}(t-s) f_{1}(s, \widehat{x}(s)) d s \\
= & \left(T_{1} \hat{x}\right)(t), \quad t \in[0, b] .
\end{aligned}
$$

Thus $T_{1}$ is $\Upsilon$-orbitally continuous.

Let $x_{1}, x_{2} \in D$ be comparable such that $x_{1} \geq x_{2}$. We have the following:

$$
\begin{aligned}
& \left\|\left(T_{1} x_{1}\right)(t)-\left(T_{1} x_{2}\right)(t)\right\| \leq \int_{0}^{t}(s-t)^{\alpha-1} \\
& \cdot\left\|\Phi_{\alpha}(t-s)\left[f_{1}\left(s, x_{1}(s)\right)-f_{1}\left(s, x_{2}(s)\right)\right]\right\| d s \\
& \leq \frac{d \varrho}{\Gamma(\alpha)} \int_{0}^{t}(s-t)^{\alpha-1} \phi\left(\left\|x_{1}(s)-x_{2}(s)\right\|\right) d s \\
& \quad \leq \frac{d \varrho b^{\alpha}}{\Gamma(\alpha+1)} \phi\left(\left\|x_{1}(s)-x_{2}(s)\right\|_{[0, b]}\right) .
\end{aligned}
$$

Since $0<\varrho<\Gamma(\alpha+1) / d b^{\alpha}$, we get that

$$
\begin{aligned}
& \left\|\left(T_{1} x_{1}\right)(t)-\left(T_{1} x_{2}\right)(t)\right\|_{C_{[0, b]}} \\
& \quad \leq \phi\left(\left\|x_{1}(s)-x_{2}(s)\right\|_{C_{[0, b]}}\right) .
\end{aligned}
$$

Hence, $T_{1}$ is partially nonlinear $D-$ contraction operator in E.

Step 2 . The operators $T_{2}, T_{3}, \ldots, T_{n}: D \longrightarrow D$ are $\Upsilon$ - orbitally continuous, nondecreasing, and partially compact operators in $E$.

First, we prove that $T_{2}, T_{3}, \ldots, T_{n}$ are mappings from $D$ to $D$. By the condition $\left(C_{3}\right)$, for any $x \in E$ such that $\|x\|_{C_{[0, b]}} \leq \tilde{r}$, we get that

$$
\begin{aligned}
\left\|\left(T_{2} x\right)(t)\right\| & \leq \frac{d}{\Gamma(\alpha)} \int_{0}^{t}(t-s)^{\alpha-1}\left\|f_{2}(s, x(s))\right\| d s \\
& \leq \frac{d N_{2} b^{\alpha}}{\Gamma(\alpha+1)} \leq r_{2} .
\end{aligned}
$$

Similarly, we have that

$$
\begin{gathered}
\left\|\left(T_{3} x\right)(t)\right\| \leq r_{3}, \\
\vdots \\
\left\|\left(T_{n} x\right)(t)\right\| \leq r_{n} .
\end{gathered}
$$

Thus, we get the following:

$$
\left\|T_{2} x\right\|_{C_{[0, b]}} \leq r_{2}, \ldots,\left\|T_{n} x\right\|_{C_{[0, b]}} \leq r_{n} .
$$

Therefore, we get that

$$
\begin{aligned}
& \left\|x_{0}-T_{2} x\right\|_{C_{[0, b]}} \leq\left\|x_{0}\right\|_{C_{[0, b]}}+\left\|T_{2} x\right\|_{C_{[0, b]}} \leq\left\|x_{0}\right\|_{C_{[0, b]}} \\
& \quad+r_{2}=\widetilde{r}_{2} \\
& \vdots \| \begin{array}{l}
\left\|x_{0}-T_{n} x\right\|_{C_{[0, b]}} \leq\left\|x_{0}\right\|_{C_{[0, b]}}+\left\|T_{n} x\right\|_{C_{[0, b]}} \leq\left\|x_{0}\right\|_{C_{[0, b]}} \\
+r_{n}=\tilde{r}_{n} .
\end{array}
\end{aligned}
$$

Hence, $T_{2}, \ldots, T_{n}$ are mappings from $D$ to $D$. By using the same method as in Step 1, we can prove $T_{2}, \ldots, T_{n}$ are $Y-$ orbitally continuous and nondecreasing $E$. Consider $C \in D$ is any arbitrary chain subset of $D$. For any $Y \in T_{2}(C)$, there is an element $x \in D$ such that $Y(t)=\left(T_{2} x\right)(t)$, for all $t \in[0, b]$. For any $t_{1}, t_{2} \in[0, b]$ such that $t_{1}<t_{2}$, we get that

$$
\begin{aligned}
\left\|Y\left(t_{2}\right)-Y\left(t_{1}\right)\right\| & \leq\left\|\left(T_{2} x\right)\left(t_{2}\right)-\left(T_{2} x\right)\left(t_{1}\right)\right\| \\
& \leq H_{1}+H_{2}+H_{3},
\end{aligned}
$$


where

$$
\begin{aligned}
& H_{1}=\| \int_{0}^{t_{1}}\left[\left(t_{2}-s\right)^{\alpha-1}-\left(t_{1}-s\right)^{\alpha-1}\right] \Phi_{\alpha}\left(t_{2}-s\right) \\
& \cdot f_{2}(s, x(s)) d s \|, \\
& H_{2}=\| \int_{0}^{t_{1}}\left(t_{1}-s\right)^{\alpha-1}\left[\Phi_{\alpha}\left(t_{2}-s\right)-\Phi_{\alpha}\left(t_{1}-s\right)\right] \\
& \cdot f_{2}(s, x(s)) d s \|, \\
& H_{3}=\left\|\int_{t_{1}}^{t_{2}}\left(t_{2}-s\right)^{\alpha-1} \Phi_{\alpha}\left(t_{2}-s\right) f_{2}(s, x(s)) d s\right\| \\
& \leq \frac{d N_{2} b^{\alpha}}{\Gamma(\alpha+1)}\left(t_{2}-t_{1}\right)^{\alpha} .
\end{aligned}
$$

Hence, when $t_{2}-t_{1} \longrightarrow 0$, we get that

$$
\mathrm{H}_{3} \longrightarrow 0
$$

and also,

$$
H_{1} \leq \frac{d N_{2}}{\Gamma(\alpha)} \int_{0}^{t_{1}}\left|\left(t_{2}-s\right)^{\alpha-1}-\left(t_{1}-s\right)^{\alpha-1}\right| d s \longrightarrow 0 .
$$

Let $t_{1}=0$ and $0<t_{2}<b$; the we get that $H_{2} \longrightarrow 0$. For $t_{1}>0$ and $\varepsilon \in\left(0, t_{1}\right)$ where $\varepsilon$ is very small, then we get that

$$
\begin{aligned}
H_{2} & \leq \| \int_{0}^{t_{1}-\varepsilon}\left(t_{1}-s\right)^{\alpha-1}\left[\Phi_{\alpha}\left(t_{2}-s\right)-\Phi_{\alpha}\left(t_{1}-s\right)\right] \\
\cdot & f_{2}(s, x(s)) d s\|+\| \int_{t_{1}-\varepsilon}^{t_{1}}\left(t_{1}-s\right)^{\alpha-1} \\
\cdot & {\left[\Phi_{\alpha}\left(t_{2}-s\right)-\Phi_{\alpha}\left(t_{1}-s\right)\right] f_{2}(s, x(s)) d s \| . } \\
& \leq \frac{N_{2}\left(t_{1}^{\alpha}-\varepsilon^{\alpha}\right)}{\alpha} \sup _{s \in\left[0, t_{1}-\varepsilon\right]}\left\|\Phi_{\alpha}\left(t_{2}-s\right)-\Phi_{\alpha}\left(t_{1}-s\right)\right\| \\
& +\frac{2 d N_{2} \varepsilon^{\alpha}}{\Gamma(\alpha+1)}
\end{aligned}
$$

By using condition $\left(C_{1}\right)$, we get that $H_{2} \longrightarrow 0$ as $t_{2}-t_{1} \longrightarrow$ 0 . Therefore, $\left\|Y\left(t_{2}\right)-Y\left(t_{1}\right)\right\| \longrightarrow 0$ as $t_{2}-t_{1} \longrightarrow 0$. Thus, $T_{2}(C)$ is equicontinuous on $[0, b]$. Also, using condition $\left(C_{3}\right)$, we get that

$$
\begin{aligned}
\|Y(t)\| & =\left\|\left(T_{2} x\right)(t)\right\| \\
& \leq \int_{0}^{t}(t-s)^{\alpha-1}\left\|\Phi_{\alpha}(t-s) f_{2}(s, x(s))\right\| d s \\
& \leq \frac{d N_{2} b^{\alpha}}{\Gamma(\alpha+1)}, \quad \forall t \in[0, b] .
\end{aligned}
$$

Hence, $T_{2}(C)$ is uniformly bounded operator in $E$ and $T_{2}$ is partially compact operator from $D$ into itself. Similarly, by doing the same steps we get that $T_{3}, \ldots, T_{n}: D \longrightarrow D$ are partially compact operators.

Step 3. Prove the existence of the $\mathrm{N}$-tupled fixed point. By condition $\left(C_{4}\right)$, we get that

$$
\begin{aligned}
\tilde{x}= & D_{*}^{\alpha} \tilde{x}(t)+A \tilde{x}(t) \\
\leq & \Psi_{\alpha}(t) \xi_{0}+\int_{0}^{t}(t-s)^{\alpha-1)} \Phi_{\alpha}(t-s) f_{1}(t, \tilde{x}(t)) d s \\
& +\int_{0}^{t}(t-s)^{\alpha-1)} \Phi_{\alpha}(t-s) f_{2}\left(t, y_{2}(t)\right) d s+\cdots \\
& +\int_{0}^{t}(t-s)^{\alpha-1)} \Phi_{\alpha}(t-s) f_{n}\left(t, y_{n}(t)\right) d s \\
= & \left(T_{1} \tilde{x}\right)(t)+\left(T_{2} y_{2}\right)(t)+\cdots+\left(T_{n} y_{n}\right)(t), \\
& \forall y_{2}, \ldots, y_{n} \in D, t \in[0, b] \quad b>0 .
\end{aligned}
$$

By applying Theorem 7, we get that the system of fractional evolution equations (19) has $\mathrm{N}$-tuple mild solution in $E_{n}$.

\section{An Application to Fractional Dynamical System}

Consider the following fractional dynamical system:

$$
\begin{aligned}
& D_{*}^{1 / 2} x_{1}(z, t)+\frac{\partial x_{1}(z, t)}{\partial z} \\
&= f_{1}\left(z, t, x_{1}(z, t)\right)+f_{2}\left(z, t, x_{2}(z, t)\right) \\
&+f_{3}\left(z, t, x_{3}(z, t)\right), \quad(z, t) \in[0,1] \times[0,1], \\
& D_{*}^{1 / 2} x_{2}(z, t)+\frac{\partial x_{2}(z, t)}{\partial z} \\
&= f_{1}\left(z, t, x_{2}(z, t)\right)+f_{2}\left(z, t, x_{3}(z, t)\right) \\
&+f_{1}\left(z, t, x_{3}(z, t)\right), \quad(z, t) \in[0,1] \times[0,1], \\
& D_{*}^{1 / 2} x_{3}(z, t)+\frac{\partial x_{3}(z, t)}{\partial z} \\
&= f_{1}\left(z, t, x_{3}(z, t)\right)+f_{2}\left(z, t, x_{1}(z, t)\right) \\
&+f_{3}\left(z, t, x_{2}(z, t)\right), \quad(z, t) \in[0,1] \times[0,1], \\
& x_{1}(0, t)=x_{1}(1, t)=x_{2}(0, t)=x_{2}(1, t)=x_{3}(0, t) \\
&= x_{3}(1, t)=0, \quad t \in[0,1], \\
& x_{1}(z, 0)=x_{2}(z, 0)=x_{3}(z, 0)=\xi_{0}(z), \quad z \in(0,1),
\end{aligned}
$$

such that $f_{2}:[0,1] \times[0,1] \times R$ is defined as

$$
f_{2}(z, t, x)= \begin{cases}5, & \text { if } x \leq 0 \\ 5+\frac{5 x}{1+2 x}, & \text { if } 0<x<2 \\ 7, & \text { if } x \geq 2\end{cases}
$$


and $f_{3}:[0,1] \times[0,1] \times R$ is defined as

$$
f_{3}(z, t, x)= \begin{cases}1, & \text { if } x \leq 0 \\ 1+2 x, & \text { if } 0<x<5 \\ 11, & \text { if } x \geq 5\end{cases}
$$

Thus $f_{2}, f_{3}$ are continuous and nondecreasing, $\left|f_{1}(z, t, x)\right| \leq$ 7 and $\left|f_{3}(z, t, x)\right| \leq 11$. Hence, condition $\left(C_{3}\right)$ is satisfied.

Theorem 10. Let the following two conditions hold:

$\left(C_{1}\right)^{\prime}$ The function $f_{1}:[0,1] \times[0,1] \times R \rightarrow R$ is continuous and there exists a constant $\varrho \in R$ with $0<$ $\varrho<\sqrt{\pi} / 2$ and $a$ D- function $\phi:[0, \infty) \longrightarrow[0, \infty)$ and satisfied the fact that $\phi(t)<r$ for any $r \in(0, \infty)$ and

$$
\begin{aligned}
0 & \leq f_{1}\left(z, t, y_{1}(z, t)\right)-f_{1}\left(z, t, y_{2}(z, t)\right) \\
& \leq \varrho \phi\left(\left|y_{1}-y_{2}\right|\right)
\end{aligned}
$$

for all $(z, t) \in[0,1] \times[0,1]$ and $y_{1}, y_{2} \in C_{[0,1]}(R)$ such that $y_{1} \geq y_{2}$.

$\left(C_{2}\right)^{\prime}$ There exists an element $\tilde{x} \in C_{[0,1]}(R) \in$ which satisfies the fact that

$$
\begin{aligned}
D_{*}^{\alpha} \tilde{x}(z, t)+\frac{\partial \tilde{x}(z, t)}{\partial z} \leq & \left.f_{1}(z, t, \tilde{x})\right)+f_{2}\left(t, y_{2}(t)\right) \\
& +f_{3}\left(t, y_{3}(t)\right), \\
\quad \forall y_{2}, y_{3} \in C_{[0,1]}(R), & \\
\tilde{x} \leq & \xi_{0}(z, 0), \quad z \in(0,1), \\
\alpha & \in(0,1), \\
t & \in[0,1] .
\end{aligned}
$$

Then the fractional dynamical system (46) has a Ntupled mild solution.

Proof. It is clear that if $X=C_{[0,1]}(R), X$ is $\Upsilon$ - orbitally complete normed linear space with $\|x(t)\|_{X}=\max _{t \in[0,1]}|x(t)|$. Let $K=\{x \in X: x \geq 0\}$; the $K$ is closed convex positive cone in $\mathrm{X}$. Thus $K$ is normal. Let $F: D(F) \longrightarrow X$, which $D(F) \subset X$, be defined as

$$
\begin{aligned}
& F y=y^{\prime}, \\
& \qquad y \in D(F)=\left\{y=X: y^{\prime} \in X, y(0)=y(1)=0\right\} .
\end{aligned}
$$

Thus we can find A-generates a $C_{0}-\operatorname{semigroup}\{S(t): t \geq 0\}$ which is defined as

$$
S(t) y(z)=y(t+z), \quad t \geq 0, \quad y \in X
$$

Hence, $\{S(t): t \geq 0\}$ is an equicontinuous $C_{0}$ - semigroup. Therefore, it is clear that $\sup _{t \in[0,1]}\|S(t)\| \leq 1$. Thus, condition $\left(C_{1}\right)^{\prime}$ holds. Define,

$$
\begin{aligned}
x_{1}(t)(z) & =x_{1}(z, t), \\
x_{2}(t)(z) & =x_{2}(z, t), \\
x_{3}(t)(z) & =x_{3}(z, t), \\
f_{1}\left(t, x_{1}(t)\right)(z) & =f_{1}\left(z, t, x_{1}(z, t)\right) ; \\
f_{2}\left(t, x_{1}(t)\right)(z) & =f_{2}\left(z, t, x_{1}(z, t)\right) ; \\
f_{3}\left(t, x_{1}(t)\right)(z) & =f_{3}\left(z, t, x_{1}(z, t)\right) ;
\end{aligned}
$$

Thus, the fractional dynamical system (46) can be written as the fractional system (19). From Theorem 9 system (46) has a $\mathrm{N}$-tupled mild solution.

\section{Conclusions}

This paper introduced a new version of Kransnoselskii fixed point theorem. This version is more general because by it we can study the $\mathrm{N}$-tupled fixed point. We applied the abstract proposed fixed point theorem to prove the existence of mild solution of the system of $\mathrm{N}$-fractional evolution equations. We gave an example of the fractional dynamical system to illustrate the abstract fixed point proposed theorem. The results are interesting and can be directly applied to some other interesting problems such as the nonlocal problems for fuzzy implicit fractional differential systems [14] and fuzzyvalued equations [15].

\section{Data Availability}

No data were used to support this study.

\section{Conflicts of Interest}

The author declared no potential conflicts on interest with respect to the research, authorship, and/or publication of this article.

\section{Acknowledgments}

The author extend his appreciation to the Deanship of Scientific Research at King Khalid University for funding this work through General Research Project under Grant no. G.R.P-98-39.

\section{References}

[1] M. Krasnoselskii, Topological Methods in the Theory of Nonlinear Integral Equations, Macmillan, New York, NY, USA, 1964.

[2] T. A. Burton, "Krasnoselskii's inversion principle and fixed points," Nonlinear Analysis, vol. 30, pp. 3975-3986, 1997.

[3] T. A. Burton and C. Kirk, "A fixed point theorem of Krasnoselskii-Schaefer type," Mathematische Nachrichten, vol. 189, pp. 23-31, 1998. 
[4] T. G. Bhaskar and V. Lakshmikantham, "Fixed point theorems in partially ordered metric spaces and applications," Nonlinear Analysis: Theory, Methods \& Applications, vol. 65, no. 7, pp. 13791393, 2006.

[5] N. V. Luong and N. X. Thuan, "Coupled fixed point theorems for mixed monotone mappings and an application to integral equations," Computers \& Mathematics with Applications. An International Journal, vol. 62, no. 11, pp. 4238-4248, 2011.

[6] B. C. Dhage, "Hybrid fixed point theory in partially ordered normed linear spaces and applications to fractional integral equations," Differential Equations \& Applications, vol. 5, no. 2, pp. 155-184, 2013.

[7] H. Yang, R. P. Agarwal, H. K. Nashine, and Y. Liang, "Fixed point theorems in partially ordered Banach spaces with applications to nonlinear fractional evolution equations," Journal of Fixed Point Theory and Applications, vol. 19, no. 3, pp. 1661-1678, 2017.

[8] M. Imdad, A. H. Soliman, B. S. Choudhury, and P. Das, "On $n$-tupled coincidence point results in metric spaces," Journal of Operators, vol. 2013, Article ID 532867, 8 pages, 2013.

[9] I. Podlubny, Fractional Differential Equations, Academic Press, San Diego, Calif, USA, 1999.

[10] K. Diethelm, N. J. Ford, and A. D. Freed, "A predictor-corrector approach for the numerical solution of fractional differential equations," Nonlinear Dynamics, vol. 29, no. 1-4, pp. 3-22, 2002.

[11] M. F. Elettreby, A. A. Al-Raezah, and T. Nabil, "Fractionalorder model of two-prey one-predator system," Mathematical Problems in Engineering, vol. 2017, Article ID 6714538, 12 pages, 2017.

[12] J. Liang and H. Yang, "Controllability of fractional integrodifferential evolution equations with nonlocal conditions," Applied Mathematics and Computation, vol. 254, pp. 20-29, 2015.

[13] Y. Zhou and F. Jiao, "Nonlocal Cauchy problem for fractional evolution equations," Nonlinear Analysis: Real World Applications, vol. 11, no. 5, pp. 4465-4475, 2010.

[14] H. V. Long and N. P. Dong, "An extension of Krasnoselskii's fixed point theorem and its application to nonlocal problems for implicit fractional differential systems with uncertainty," Journal of Fixed Point Theory and Applications, vol. 20, no. 1, article 37, 2018.

[15] N. T. K. Son and N. P. Dong, "Asymptotic behavior of $C^{0}-$ solutions of evolution equations with uncertainties," Journal of Fixed Point Theory and Applications, vol. 20, no. 4, article 153, 2018. 


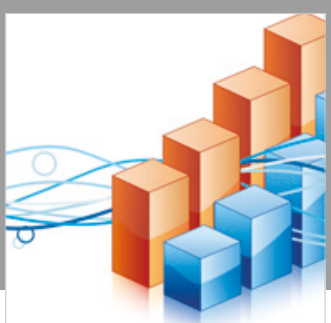

Advances in

Operations Research

\section{-n-m}
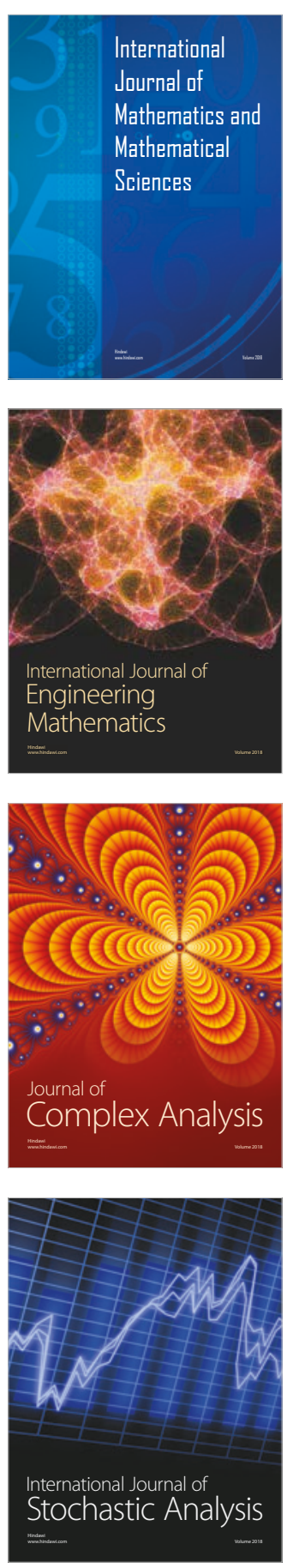
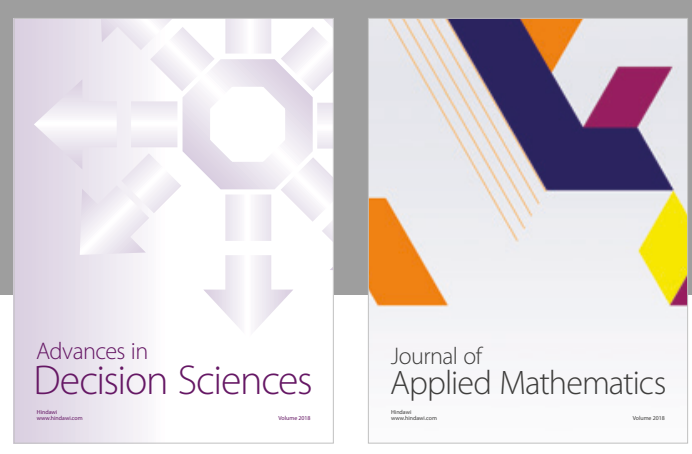

Journal of

Applied Mathematics
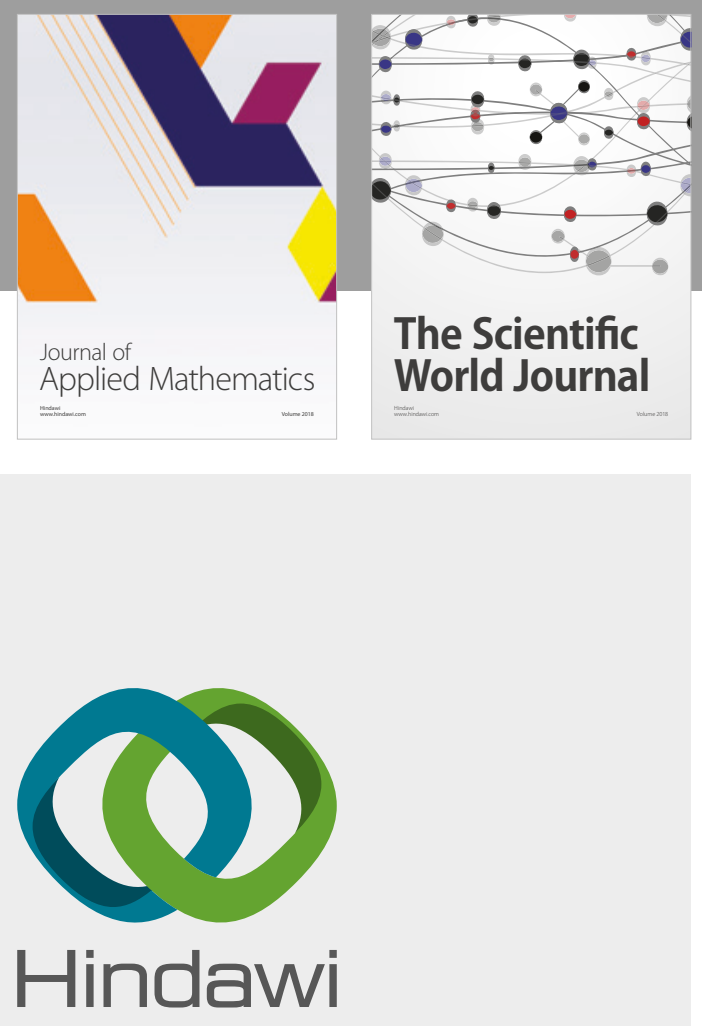

Submit your manuscripts at

www.hindawi.com

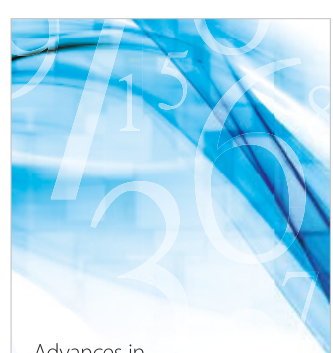

Advances in
Numerical Analysis
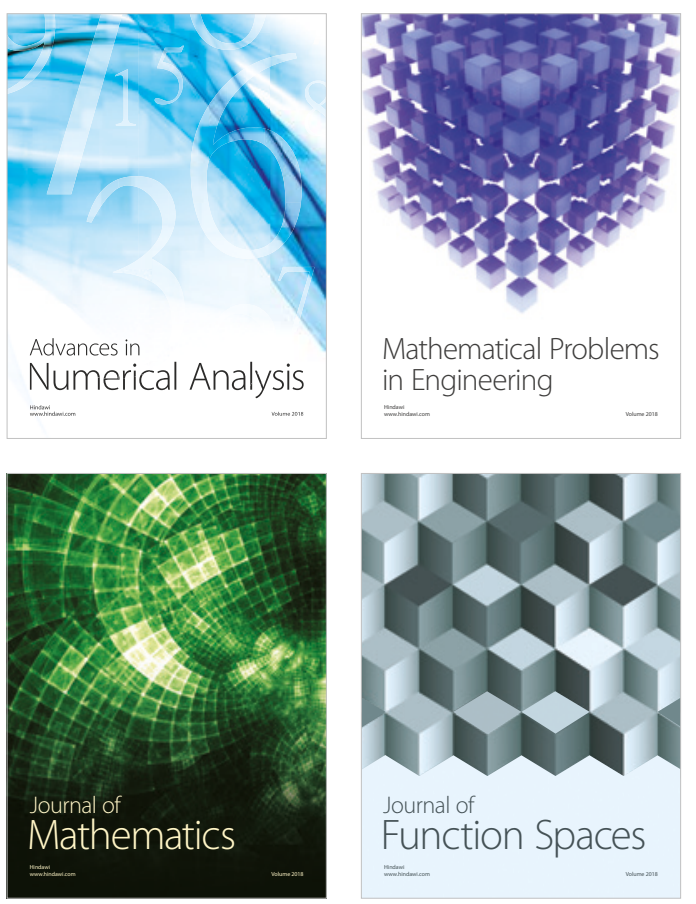

Mathematical Problems in Engineering

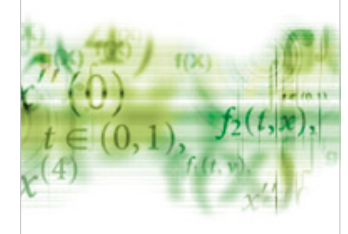

International Journal of

Differential Equations

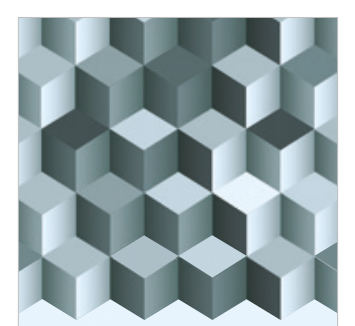

Journal of

Function Spaces

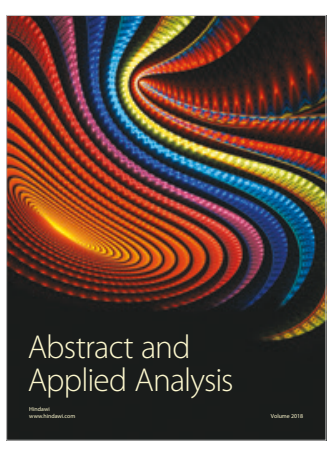

The Scientific

World Journal

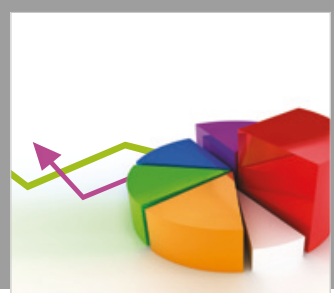

Journal of

Probability and Statistics
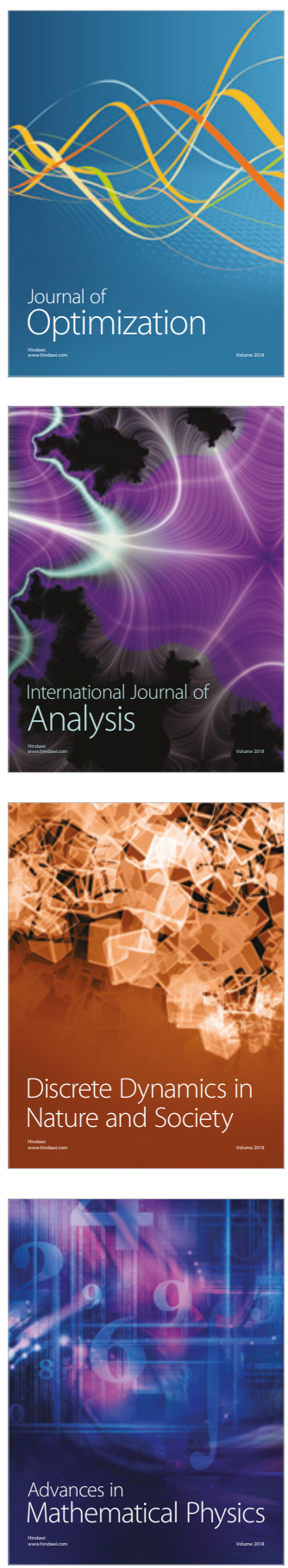